\title{
Nature du caractère épaississant de certaines souches de Streptococcus thermophilus : étude préliminaire
}

\author{
G. GIRAFFA* et J.-L. BERGÈRE \\ INRA, Laboratoire de la chaire de Technologie, INA-PG, 78850 Thivernal-Grignon, France \\ * Actuellement: Instituto Sperimentale Lattiero Caseario, Lodi, Italie
}

\section{Résumé}

Trois souches épaississantes de $S$. thermophilus et une non épaississante ont été comparées après culture, soit dans du lait à différentes températures, soit dans d'autres milieux : lait supplémenté ou dialysé, lactosérum et milieu Elliker à $44^{\circ} \mathrm{C}$.

Les souches épaississantes se caractérisent par une augmentation de la viscosité qui ne se manifeste que dans le lait mais pas dans les autres milieux. Ces souches produisent plus de polysaccharides solubles que la souche non épaississante mais les quantités sont faibles $(\leqslant 40 \mathrm{mg} / \mathrm{l})$ et pas en rapport avec les variations de viscosité observées. A $30^{\circ} \mathrm{C}$, les souches épaississantes produisent de 4 à 8 fois plus de glucides insolubles que la souche non épaississante mais, dans les autres cas, les différences sont beaucoup plus faibles. Quelles que soient les conditions de culture dans le lait, les profils électrophorétiques des caséines sont les mêmes pour les quatre souches.

A partir de ces résultats il est difficile de conclure sur la nature possible du composé responsable des propriétés épaississantes de ces souches de $S$. thermophilus mais plusieurs hypothèses sont émises.

Mots clés : S, thermophilus - Souches épaississantes - Viscosité - Polysaccharides Protéolyse.

\section{Summary}

Nature of the viscous character of some Streptococcus thermophilus strains : a preliminary investigation

Three ropy and one non ropy strains of $S$. thermophilus were compared after growth either in skim milk at various temperatures or in supplemented or dialyzed milk, whey and Elliker medium at $44{ }^{\circ} \mathrm{C}$. Ropy strains exhibited increase in relative viscosity only in milk media but not in the other ones. They produced more soluble polysaccharides than the non ropy strain but the amounts were low $(\leqslant 40 \mathrm{mg} / \mathrm{l})$ and not related to the viscosity. At $30^{\circ} \mathrm{C}$, ropy strains produced 4 to 8 times more insoluble glucides than the non ropy strain but in the other cases the differences were generally 
lower. Whatever the culture conditions in milk, electrophoretic pattern of caseins were quite similar for the four strains.

From this results it is difficult to conclude on the nature of the viscous character of $S$. thermophilus but some hypothesis are postulated.

Key words : S. thermophilus - Ropy strains - Viscosity - Polysaccharides - Proteolysis.

\section{Introduction}

Certaines souches de Streptococcus thermophilus qualifiées d'épaississantes ou de filantes sont utilisées en plus, ou à la place des souches normales dans la fabrication de certains yaourts, notamment les yaourts brassés, parce qu'elles confèrent des propriétés rhéologiques particulières au gel résultant de leur activité acidifiante (MARTENS, 1972; GALESLOOT et HASSING, 1973 ; VANDERPOORTEN et Waes, 1972, Boulllanne et Desmazeaud, 1980). En culture sur lait à l'état pur, ces souches donnent des gels plus visqueux (BoullLanNe et Desmazeaud, 1980 ; SchellhaAss, 1983) qui seraient aussi plus thixotropiques et moins sensibles à la synérèse que ceux obtenus avec des souches normales (ShellhaAss, 1983). Mais ces souches n'ont encore été que très peu étudiées et, à notre connaissance, le mécanisme par lequel elles modifient les propriétés du gel qui viennent d'être mentionnées n'a pas encore été élucidé. On a coutume de l'attribuer à la production de polysaccharides par analogie avec ce qui a été trouvé en général chez les bactéries qui élaborent des produits visqueux dans les milieux de culture ou certains produits naturels (DUNICAN et Seely, 1965 ; SlODKI et CADmus, 1978), et plus particulièrement chez les bactéries lactiques qui provoquent des modifications de viscosité du lait (RANGANATHAN et al., 1979) ou de certains laits fermentés (LA RIvière et al., 1967 ; Groux, 1973 ; TAmime et Robinson, 1978 ; OdA et al., 1983 ; MANCA dE NADRA et al., 1985).

Chez $S$. thermophilus la production d'un polysaccharide composé de glucose et de galactose déjà mentionnée à propos d'une activité antitumorale (TsuchiYa et al., 1981) a été montrée chez des souches filantes isolées de yaourt (SchellhaAss, 1983). Cependant il n'a pas été prouvé que c'est bien ce polysaccharide qui intervient dans les modifications de propriété des gels de lait mentionnées ci-dessus; les quantités produites n'étant pas indiquées et surtout la présence de ce composé n'ayant pas été recherchée chez des souches normales, il n'est pas possible de conclure qu'il s'agit d'une particularité propre à ces souches filantes.

En vue d'une étude plus approfondie sur la nature, l'expression et la stabilité du caractère épaississant de ces $S$. thermophilus particuliers, nous avons effectué un travail préliminaire sur quelques souches épaississantes en comparaison avec une souche normale. La production de polysaccharides a été recherchée en fonction de facteurs qui ont déjà été mentionnés dans les travaux cités ci-dessus comme susceptibles de la favoriser chez d'autres bactéries. D'autre part, l'augmentation de la viscosité pouvant provenir d'une modification des caséines par des enzymes telles que des protéases, l'état des protéines du lait des différentes cultures a été examiné par l'électrophorèse. 


\section{Matériels et méthodes}

\section{- Souches utilisées}

Trois souches de $S$. thermophilus (CNRZ 404, 783 et 784) classées comme épaississantes (Bouillanne et Desmazeaud, 1980) et une souche normale (CNRZ 388) ont été utilisées. Une souche de L. bulgaricus non visqueuse (CNRZ 398) a également été essayée en association. Ces souches provenaient de la collection du CNRZ.

\section{- Conditions de culture}

Les souches d'origine conservées à l'état lyophilisé étaient remises en culture et repiquées plusieurs fois (voir résultats) dans du lait écrémé stérile avant de constituer un stock de cultures sur lait (inoculum $5 \%$ ) conservées à $-30^{\circ} \mathrm{C}$. Au fur et à mesure des essais ces cultures étaient décongelées et incubées $16 \mathrm{~h}$ à $44{ }^{\circ} \mathrm{C}$. Elles étaient ensuite inoculées à raison de $5 \%$ dans $200 \mathrm{ml}$ de milieu puis incubées à $44{ }^{\circ} \mathrm{C}$ ou à la température indiquée dans les résultats.

Le milieu de culture utilisé était soit du lait écrémé reconstitué à partir de poudre $(100 \mathrm{~g} / \mathrm{l})$, soit du lait écrémé additionné de saccharose $(25 \mathrm{~g} / \mathrm{l})$ ou de glucose + galactose $(25 \mathrm{~g} / \mathrm{l}$ de chaque) ou d'extrait de levure Difco $(0,5$ ou $5 \mathrm{~g} / \mathrm{l})$, soit de milieu Elliker Difco contenant du glucose, du lactose et du saccharose (5 g/l de chaque) (Eluiker et al., 1956), soit de lactosérum reconstitué à partir de poudre $(10 \mathrm{~g} / \mathrm{l})$ et filtré, soit enfin de lait écrémé dialysé additionné de Casamino acids (Difco vitamin free, $5 \mathrm{~g} / 1$ ) (ScHelLHAASS, 1983). Ils étaient stérilisés à $110^{\circ} \mathrm{C}$ pendant $10 \mathrm{mn}$.

Après $24 \mathrm{~h}$ d'incubation, chaque culture était ainsi fractionnée : $9 \mathrm{ml}$ servaient à la mesure de la viscosité, $10 \mathrm{ml}$ à la mesure du $\mathrm{pH}, 100 \mathrm{ml} \mathrm{au}$ dosage des polysaccharides et $10 \mathrm{ml}$ étaient réservés pour l'électrophorèse dans le cas des cultures sur lait. En outre, dans le cas des cultures en association avec le lactobacille, il a été vérifié par dénombrement au microscope que $S$. thermophilus s'était bien développé. Pour toutes les autres cultures la croissance était seulement appréciée indirectement par la mesure du $\mathrm{pH}$.

\section{- Mesure de la viscosité}

La viscosité des cultures était mesurée à $44{ }^{\circ} \mathrm{C}$ par détermination directe des contraintes de cisaillement sur $9 \mathrm{ml}$ de culture ou de milieu non ensemencé, à l'aide d'un viscosimètre à cylindres coaxiaux (Rotovisco RV2 Haake, tête de mesure NV) à une vitesse de déformation de 10 à $100 \mathrm{mn}^{-1}$. Elle était exprimée en mPa.s.

\section{- Extraction et dosage des polysaccharides}

La teneur en glucides totaux solubles et insolubles était déterminée par la méthode de Dubois et al. (1956) et exprimée arbitrairement en mg de glucose.

Les fractions soluble et insoluble étaient préparées de la manière suivante. La culture (ou le lait non inoculé) était diluée au demi avec de l'eau distillée 
après que le $\mathrm{pH}$ ait été ajusté à 7,5 avec de la soude puis la caséine était hydrolysée par de la Pronase Boehringer (rapport enzyme substrat 1/100, $44^{\circ} \mathrm{C}, 16 \mathrm{~h}$ ) en présence de chloroforme (LA RIVIÈRE et al., 1967) pour inhiber la croissance des cellules ; après un réajustement éventuel au début de l'incubation le $\mathrm{pH}$ restait stable pendant toute la durée de l'incubation. Le mélange était ensuite centrifugé à $6000 \mathrm{~g}$ pendant $20 \mathrm{mn}$ à $4{ }^{\circ} \mathrm{C}$ puis le culot était remis en suspension dans $100 \mathrm{ml}$ d'eau distillée et centrifugé dans les mêmes conditions. Le mélange des deux surnageants était précipité à l'éthanol $(60 \%$ $\mathrm{v} / \mathrm{v})$. Le précipité collecté par centrifugation $\left(6000 \mathrm{~g}, 4^{\circ} \mathrm{C}\right)$ était redissous en eau distillée et dialysé à $4{ }^{\circ} \mathrm{C}$ pendant $48 \mathrm{~h}$ avec plusieurs changements d'eau, il constituait la fraction dite soluble. Le culot obtenu après la deuxième centrifugation était dialysé de la même manière et constituait la fraction dite insoluble (cellules + polysaccharide insoluble éventuel).

Pour les cultures effectuées dans des milieux autres que le lait, le même processus était suivi à l'exclusion de l'incubation en présence de Pronase.

\section{- Electrophorèse}

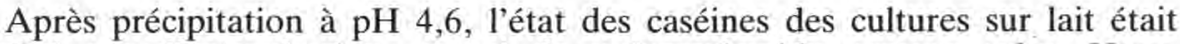
examiné par électrophorèse en gel de polyacrylamide agarose selon URIEL (1966) et électrophorèse en présence de SDS dans un gel de polyacrylamide à la concentration de $16 \%$ ou en gradient linéaire (16-28\%) selon la technique de Laemmli (1970) modifiée par Trieu-Cuot et Gripon (1981). Les protéines étaient colorées au bleu de Coomassie.

\section{Résultats}

\section{A. Evolution de la viscosité des cultures en fonction du nombre de transferts sur lait}

La viscosité des premières cultures sur lait effectuées à partir des souches lyophilisées étant très faible (fig. 1), son évolution a été suivie au cours de plusieurs transferts successifs sur lait.

Comme on peut le constater d'après cette figure, la viscosité à $24 \mathrm{~h}$ augmentait progressivement jusqu'au $6^{c}$ piquage mais plus au-delà. Le $\mathrm{pH}$ à 24 h était resté compris entre 4,8 et 4,9 pour la souche normale alors que dans le cas des souches épaississantes il avait diminué régulièrement du $1^{\mathrm{er}}$ au $6^{\mathrm{e}}$ transfert de 4,5 à 4,2-4,3 (souches 783 et 404) et de 5,2 à 4,8 (souche 784). En outre, on a observé que même au bout de quelques jours, les cultures sur lait des souches épaississantes n'exsudaient pas de sérum, ou en tout cas beaucoup moins que la souche normale, mais aucune mesure n'a été faite. Pour chaque souche épaississante la différence de viscosité par rapport à la souche normale commençait à se manifester vers $\mathrm{pH} 5,0-5,2$, c'est-à-dire en même temps que débutait la gélification du lait sous l'effet de l'acidification puis elle allait en s'amplifiant au fur et à mesure de l'incubation et de la diminution du $\mathrm{pH}$. Après avoir atteint un maximum, suivant les souches et les essais, la viscosité restait à peu près stable ou bien commençait à diminuer mais à $24 \mathrm{~h}$ elle restait toujours supérieure à celle des cultures de la souche normale. Comme 
dans la suite des essais, il n'était pas possible de suivre systématiquement l'évolution de toutes les cultures, il a été choisi de les arrêter après $24 \mathrm{~h}$ d'incubation.

Pour tous les essais qui suivent, l'inoculum provenait des cultures obtenues au $7^{\mathrm{e}}$ transfert et conservées comme il est indiqué dans les méthodes.

\section{B. Teneur en polysaccharides et viscosité des cultures effectuées dans différentes conditions}

La viscosité des cultures de la souche 784 variant beaucoup, seuls ont été rapportés les résultats se rapportant à des cultures de viscosité nettement supérieure à celles de la souche normale.

\section{Cultures sur lait effectuées à différentes températures}

Les teneurs en polysaccharides des cultures étaient relativement faibles et ne variaient pas significativement avec la température d'incubation sauf à $30^{\circ} \mathrm{C}$ où elles étaient nettement plus élevées pour les deux souches épaississantes (tabl. 1). Cette augmentation ne portait que sur la fraction insoluble qui était alors de 4 à 8 fois supérieure à celle trouvée pour la souche normale. Par contre aux autres températures, les souches épaississantes se distinguaient de la souche normale plutôt par la fraction soluble mais les quantités produites étaient très faibles.

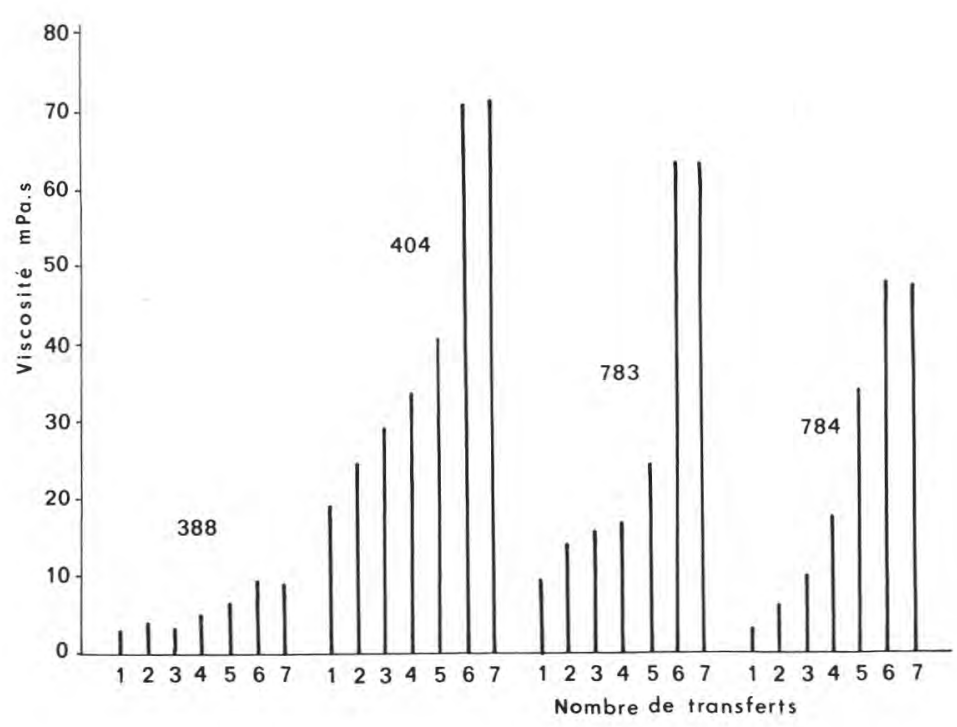

Fig. 1

Evolution de la viscosité des cultures en fonction du nombre de transferts sur lait.

Viscosity of milk cultures after serial transfers. 


\section{TABLeaU 1}

Effet de la température d'incubation sur la viscosité et la teneur en polysaccharides des cultures sur lait

Effect of incubation temperature on the amount of polysaccharide and viscosity of milk cultures

\begin{tabular}{|c|c|c|c|c|}
\hline \multirow{2}{*}{ Souche } & \multirow{2}{*}{$\begin{array}{c}\text { Température } \\
{ }^{\circ} \mathrm{C}\end{array}$} & \multirow{2}{*}{$\begin{array}{c}\text { Viscosité } \\
\mathrm{mPa} \cdot \mathrm{s}\end{array}$} & \multicolumn{2}{|c|}{ Teneur en polysaccharides $(\mathrm{mg} / \mathrm{l})^{* *}$} \\
\hline & & & solubles & insolubles \\
\hline \multirow{4}{*}{388} & 30 & 3,4 & 12 & 22 \\
\hline & 35 & 6,0 & 5 & 30 \\
\hline & 40 & 9,0 & 0 & 25 \\
\hline & 44 & 3,0 & 3 & 27 \\
\hline \multirow{4}{*}{783 * } & 30 & 5,0 & 32 & 87 \\
\hline & 35 & 18 & 30 & 16 \\
\hline & 40 & 38 & 49 & 42 \\
\hline & 44 & 41 & 14 & 61 \\
\hline \multirow{4}{*}{$404 *$} & 30 & 11 & 9 & 182 \\
\hline & 35 & 34 & 12 & 45 \\
\hline & 40 & 48 & 21 & 34 \\
\hline & 44 & 59 & 18 & 52 \\
\hline
\end{tabular}

* Souches épaississantes

* Ropy strains

** Les quantités trouvées pour le lait non inoculé, respectivement 7,6 et $1,3 \mathrm{mg} / 1$ pour les fractions soluble et insoluble ont été défalquées des résultats.

** The amounts of polysaccharides in non-inoculated milk, respectively 7.6 and $1.3 \mathrm{mg} / 1$ for soluble and unsoluble fractions were deducted.

D'autre part, les quantités trouvées n'étaient pas en rapport avec la variation de viscosité des cultures (tabl. 1). A $30^{\circ} \mathrm{C}$, c'était d'ailleurs même l'inverse. Cependant il est possible qu'à cette dernière température la viscosité n'ait pas encore atteint son maximum à $24 \mathrm{~h}$ en raison d'une croissance plus lente.

\section{Cultures sur lait supplémenté}

Les quantités de polysaccharides solubles variaient assez peu en fonction du supplément ajouté au lait et, bien qu'un peu supérieures chez les souches épaississantes, elles restaient très faibles et ne dépassaient pas $40 \mathrm{mg} / \mathrm{l}$ (tabl. 2). Les quantités de glucides insolubles ne variaient pas beaucoup plus mais atteignaient des valeurs plus élevées qui pouvaient dépasser $100 \mathrm{mg} / 1$. Ces variations allaient certainement de pair avec des augmentations de la quantité de cellules résultant de l'apport de nutriments supplémentaires. On a constaté en effet que l'addition d'extrait de levure entraînait un abaissement du $\mathrm{pH}$ des cultures de 0,2 à 0,6 unité par rapport à celui des cultures en lait seul. D'ailleurs dans les cultures de la souche normale, les teneurs en glucides insolubles augmentaient dans les mêmes proportions que dans celles des souches épaississantes. 
Les viscosités ne suivaient pas les variations de teneurs en polysaccharides à part peut-être chez la souche 404 pour laquelle les variations étaient les plus faibles. Chez la souche 784, l'addition d'extrait de levure à $0,5 \mathrm{~g} / 1$ avait un effet marqué sur la viscosité et la teneur en glucides insolubles vraisemblablement par une stimulation de la croissance car c'est la souche pour laquelle les variations de $\mathrm{pH}$ étaient les plus marquées, son développement sur lait étant généralement plus lent et plus limité que celui des deux autres souches.

\section{TABLEAU 2}

Viscosité et teneur en polysaccharides des cultures en fonction des composés ajoutés au lait

Viscosity and amounts of polysaccharide in supplemented milk cultures

\begin{tabular}{|c|c|c|c|c|c|}
\hline \multirow{2}{*}{ Souche } & \multirow{2}{*}{\multicolumn{2}{|c|}{$\begin{array}{c}\text { Composé ajouté } \\
\mathrm{g} / 1\end{array}$}} & \multirow{2}{*}{$\begin{array}{c}\text { Viscosité } \\
\mathrm{mPa} . \mathrm{s}\end{array}$} & \multicolumn{2}{|c|}{ Teneur en polysaccharides $(\mathrm{mg} / \mathrm{l}){ }^{* *}$} \\
\hline & & & & solubles & insolubles \\
\hline \multirow{5}{*}{388} & - & & 3 & 3 & 27 \\
\hline & E. levure & 0,5 & 17 & 0 & 20 \\
\hline & E. levure & 5,0 & 24 & 7 & 74 \\
\hline & Saccharose & 25 & 14 & 1 & 97 \\
\hline & Gluc. + gal. & $25+25$ & 9 & 22 & 19 \\
\hline \multirow{5}{*}{$783 *$} & - & & 41 & 14 & 61 \\
\hline & E. levure & 0,5 & 30 & 40 & 63 \\
\hline & E. levure & 5,0 & 29 & 37 & 123 \\
\hline & Saccharose & 25 & 23 & 29 & 3 \\
\hline & Gluc. + gal. & $25+25$ & 22 & 39 & 48 \\
\hline \multirow{3}{*}{$784 *$} & 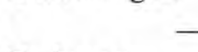 & & 32 & 12 & 33 \\
\hline & E. levure & 0,5 & 56 & 10 & 108 \\
\hline & E. levure & 5,0 & 31 & 39 & 139 \\
\hline \multirow{5}{*}{$404 *$} & 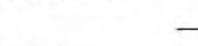 & & 59 & 18 & 52 \\
\hline & E. levure & 0,5 & 48 & 15 & 93 \\
\hline & E. levure & 5,0 & 38 & 21 & 66 \\
\hline & Saccharose & 25 & 37 & 3 & 70 \\
\hline & Gluc. + gal. & $25+25$ & 22 & 17 & 13 \\
\hline
\end{tabular}

* Souches épaississantes.

* Ropy strains.

** Valeurs défalquées des quantités trouvées pour les mêmes milieux non inoculés.

** The amounts found in the same non-inoculated media were deducted.

\section{Culture en association avec un lactobacille non épaississant}

La présence de la souche de $L$. bulgaricus avait un effet négatif sur la viscosité et la teneur en polysaccharides solubles des cultures des souches épaississantes (tabl. 3 et 1) bien que leur croissance n'ait pas été inhibée d'après les comptages au microscope. 


\section{TABLEAU 3}

Teneur en polysaccharides et viscosité des cultures de $\mathrm{S}$. thermophilus associées à un lactobacille non épaississant

Amounts of polysaccharide and viscosity of milk-cultures of $\mathrm{S}$. thermophilus associated with a non-ropy Lactobacillus

\begin{tabular}{c|c|c|c}
\hline \multirow{2}{*}{ Souches } & $\begin{array}{c}\text { Viscosité } \\
\text { mPa.s }\end{array}$ & \multicolumn{2}{|c}{ Teneur en polysaccharides $(\mathrm{mg} / 1)^{* *}$} \\
\cline { 2 - 4 } & 31 & solubles & insolubles \\
\hline $388+398^{*}$ & 31 & 0 & 277 \\
$783+398$ & 41 & 0 & 195 \\
$404+398$ & 31 & 27 & 103 \\
398 & 28 & 145 \\
\hline
\end{tabular}

* L. bulgaricus CNRZ 398.

** Valeurs défalquées des quantités trouvées pour les mêmes milieux non inoculés.

** The amounts found in the same non-inoculated media were deducted.

L'augmentation importante de la teneur en glucides insolubles résultait vraisemblablement de l'augmentation de cellules due à la présence simultanée des deux espèces ainsi qu'aux phénomènes de stimulation mutuelle bien connus chez ces deux bactéries. C'est d'ailleurs chez la souche non épaississante (388) que l'effet était le plus marqué. Le pH des cultures était de 3,8 pour les trois associations, 3,9 pour L. bulgaricus seul, voisin de 4,3 pour $S$. termophilus 783 et 404 seuls et 4,8 pour $S$. thermophilus 388 seul.

\section{Culture dans des milieux autres que le lait}

Etant donné la difficulté de dosage des polysaccharides dans le lait complet, nous avions cherché à voir quel était le comportement des souches étudiées dans trois milieux plus faciles à manipuler : du lactosérum, du lait dialysé et un milieu de culture habituel de laboratoire (milieu Elliker).

Les quatre souches étaient transférées deux fois dans chacun de ces milieux avant de procéder aux mesures, ceci afin d'éviter que le lait apporté avec le premier inoculum n'interfère.

La viscosité qui était de $1,4 \mathrm{mPa}$.s avant incubation restait ensuite constante ou atteignait au plus $1,5 \mathrm{mPa}$.s après $24 \mathrm{~h}$ aussi bien avec la souche normale qu'avec les souches épaississantes. Les teneurs en glucides solubles étaient inférieures ou égales à $1 \mathrm{mg} / \mathrm{l}$ dans le lait dialysé et le lactosérum, et ne différaient pas non plus entre souches dans le milieu Elliker à part le cas de 783 (tabl. 4). Les teneurs en glucides insolubles étaient un peu supérieures pour deux souches, à la fois en Elliker et en lactosérum, mais elles étaient les mêmes pour les quatre souches cultivées en lait dialysé.

Afin de vérifier si ces résultats étaient dus à une perte du pouvoir épaississant des souches au cours de leur passage sur ces différents milieux, nous les avons réinoculées immédiatement dans du lait et avons mesuré la 
viscosité à $24 \mathrm{~h}$. Ce n'était généralement pas le cas, car les viscosités étaient respectivement de 110, 62 et $104 \mathrm{mPa} . \mathrm{s}$ (404), 55,16 et $47 \mathrm{mPa} . \mathrm{s}$ (784) suivant que l'inoculum provenait de milieu Elliker, de lactosérum ou de lait dialysé.

\section{TABLEAU 4}

Teneur en polysaccharides et viscosité des cultures de S. thermophilus effectuées en milieu Elliker, lactosérum et lait dialysé

Amounts of polysaccharide and viscosity of milk-cultures of $\mathrm{S}$. thermophilus in Elliker medium, whey and dialyzed milk

\begin{tabular}{|c|c|c|c|}
\hline \multirow{2}{*}{ Souches } & \multirow{2}{*}{ Milieu } & \multicolumn{2}{|c|}{ Teneur en polysaccharides $(\mathrm{mg} / \mathrm{l}) * *$} \\
\hline & & solubles & insolubles \\
\hline 388 & $\begin{array}{l}\text { E } \\
\text { LS } \\
\text { LD }\end{array}$ & $\begin{array}{r}15 \\
1 \\
1\end{array}$ & $\begin{array}{l}50 \\
23 \\
23\end{array}$ \\
\hline $783 *$ & $\begin{array}{l}\text { E } \\
\text { LS } \\
\text { LD }\end{array}$ & $\begin{array}{r}35 \\
0 \\
2\end{array}$ & $\begin{array}{l}21 \\
10 \\
24\end{array}$ \\
\hline $784 *$ & $\begin{array}{l}\text { E } \\
\text { LS } \\
\text { LD }\end{array}$ & $\begin{array}{r}16 \\
0 \\
1\end{array}$ & $\begin{array}{l}12 \\
10 \\
23\end{array}$ \\
\hline $404 *$ & $\begin{array}{l}\text { E } \\
\text { LS } \\
\text { LD }\end{array}$ & $\begin{array}{r}16 \\
0 \\
1\end{array}$ & $\begin{array}{l}54 \\
24 \\
24\end{array}$ \\
\hline \multicolumn{4}{|c|}{$\begin{array}{l}\text { * Souches épaississantes. } \\
\text { * Ropy strains. } \\
\text { Valeurs défalquées de la quantité de polysaccharide trouvée dans les milieux non ensemencés lorsque celle-ci } \\
\text { nétait pas nulle. } \\
\text { E : Elliker, LS : lactosérum et LD : lait dialysé. } \\
\text { The amounts of polysaccharide found in non-inoculated media were deducted. } \\
\text { E : Elliker, LS : whey and LD : dialyzed milk. }\end{array}$} \\
\hline
\end{tabular}

\section{Examen électrophorétique de l'état des caséines dans les différentes cultures}

Toutes les cultures sur lait additionné ou non d'un supplément ont été examinées par électrophorèse. Quelles que soient les conditions de culture, aucune différence particulière n'a été mise en évidence entre les quatre souches étudiées, que les protéines du lait soient séparées selon leur charge ou bien selon leur poids moléculaire. La figure 2 en donne un exemple, les profils des souches 388,404 et 783 cultivées à $44{ }^{\circ} \mathrm{C}$ sont semblables et ne diffèrent pas de celui obtenu avec le lait stérile non inoculé. Si des différences existent, elles sont mineures et nécessiteraient pour être mises en évidence d'effectuer des cultures sur lait peu ou pas chauffé, celui-ci permettant d'obtenir des profils plus nets avec des bandes de caséine mieux séparées, et d'utiliser éventuellement d'autres techniques. 


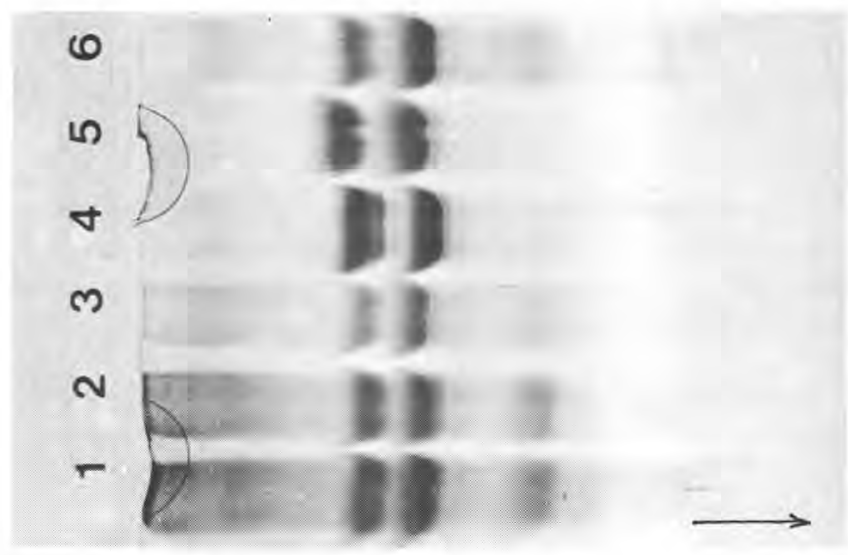

Fig. 2

Profil électrophorétique des protéines $d u$ lait en gel de polyacrylamide (16\%) en présence de SDS.

1 : souche $388 ; 2$ : souche $404 ; 3$ : souche $783 ; 4$ : caséine entière; 5 : lait «low heat »; 6: lait stérile non ensemencé.

Electrophoretic profiles and milk proteins after SDS - PAGE (16\%).

1 : strain $388 ; 2$ : strain $404 ; 3:$ strain $783 ; 4:$ whole casein ; $5:$ :low-heat» milk;

6 : non-inoculated sterile milk.

\section{Discussion}

Les souches de $S$. thermophilus utilisées dans ce travail sont bien des souches dites épaississantes, c'est-à-dire capables de produire un gel de lait plus visqueux que les souches courantes comme cela avait été montré précédemment (Bouillanne et Desmazeaud, 1980).

Cette augmentation de la viscosité ne se manifeste que dans le lait qui n'est pas débarrassé de ses caséines, soit que le composé qui en est responsable ne soit pas produit dans les autres milieux, soit qu'en raison d'une concentration trop faible et (ou) de ses propriétés, il ne soit pas capable de modifier la viscosité d'un milieu aqueux en absence de caséine. Dans le lait lui-même, l'augmentation de la viscosité propre à ces souches particulières ne se manifeste d'ailleurs qu'à partir du moment où les micelles de caséines commencent à être déstabilisées sous l'effet de l'acide produit, puis elle s'accentue en même temps que le $\mathrm{pH}$ s'abaisse, ceci au moins pendant un certain temps. Cela est sûrement en rapport avec la croissance des bactéries et la nécessité d'atteindre une certaine concentration du composé à partir de laquelle il agit en synergie avec l'épaississement dû à la gélification. Mais il est vraisemblable que l'augmentation de viscosité soit aussi due à une interaction plus complexe entre le composé en cause et les caséines et soit ainsi fonction du $\mathrm{pH}$. Comme ce dernier évolue, la viscosité mesurée sur les cultures n'est donc pas nécessairement en rapport étroit avec la concentration du composé qui en est responsable. 
Quoiqu'il en soit, on pouvait s'attendre à ce que des variations de conditions de culture aient un effet marqué à la fois sur la viscosité et la production de polysaccharides et permettent d'avancer qu'il y a bien un lien de cause à effet entre ces deux phénomènes. Or l'ensemble des résultats obtenus ne permet pas de montrer que l'augmentation de viscosité du lait provoquée par les souches épaississantes de $S$. thermophilus est bien due à la production d'un polysaccharide soluble. En effet si les quantités produites par ces souches sont en général supérieures à celles trouvées pour la souche normale, elles ne dépassent pas $40 \mathrm{mg} / \mathrm{l}$ et sont donc relativement faibles par rapport à ce qui a été observé chez d'autres bactéries lactiques affectant la viscosité du lait coagulé (LA Rivière et al., 1967; CERnING et al., 1986). La production de polysaccharides solubles par $S$. thermophilus ne varie pas beaucoup suivant les essais et ne parait pas induite par le saccharose comme c'est le cas chez de nombreuses autres bactéries dont des streptocoques, en particulier $S$. salivarius très apparenté à $S$. thermophilus, ni stimulée par une croissance à température sub-optimale (NIVEN et al., 1941 et 1946; DAIN et al., 1956; DuNICAN et Seely, 1965 ; Ranganathan et al., 1979 ; Sutherland, 1982).

Les seuls effets marqués concernent les glucides insolubles dont la quantité était notablement augmentée en présence d'extrait de levure et surtout en culture à basse température. Cependant comme la croissance dans le lait n'a pas été mesurée, il est d'ailleurs difficile de le faire avec précision, il n'est pas possible de dire si la totalité de ces glucides provient uniquement de constituants cellulaires (en particulier pariétaux) ou s'il s'agit, au moins en partie, d'un polysaccharide exocellulaire insoluble (par exemple capsulaire). Les quantités trouvées ne sont pas incompatibles avec la première hypothèse si on se rapporte à la quantité de cellules présentes. Les différences observées seraient alors seulement dues à la présence d'une plus ou moins grande quantité de cellules. C'est peut-être le cas pour les cultures en présence d'extrait de levure d'autant plus qu'on observe le même phénomène pour la souche normale. Par contre à $30^{\circ} \mathrm{C}$, il est plus vraisemblable que les glucides dosés proviennent en grande partie d'un polysaccharide insoluble plutôt que des cellules ellesmêmes. La présence de polysaccharides insolubles présents en quantité importante, en partie sous forme de capsule, a d'ailleurs déjà été montrée chez des leuconostoc et des streptocoques (Brooker, 1979 ; Fukushima et al., 1982 ; Sutherland, 1982). Mais on peut se demander alors si ce type de polysaccharide intervient bien dans l'augmentation de viscosité due aux souches épaississantes de $S$. thermophilus puisque c'est à cette température qu'elle était la plus basse, à moins qu'elle soit justement plus faible en raison d'un excès de polysaccharide et d'un gel moins rigide à cette température comme l'a avancé SchellhaAsS (1983).

Cependant si on admet que dans les autres conditions de culture, une partie de la fraction insoluble est bien constituée de ce polysaccharide, les quantités trouvées restent relativement faibles, même si on y ajoute la fraction soluble, et permettent difficilement d'expliquer les modifications de propriétés du caillé observées. On est donc conduit à émettre les hypothèses suivantes :

- ou bien le composé responsable est bien un (ou des) polysaccharide qui pourrait être en partie insoluble (mélange de formes ou de types différents), mais il faudrait qu'il ait des propriétés très particulières pour avoir un effet à ces concentrations. Il pourrait s'agir des polymères de glucose-galactose 
identifiés par Tsuchiya (1981) ou par SchellhaAss (1983) à partir de cultures de $S$. thermophilus sur lait dialysé, mais malheureusement leurs propriétés n'ont pas été étudiées, et nous n'avons pas trouvé nous-mêmes de production de polysaccharide soluble dans ce milieu ;

- ou bien le polysaccharide responsable a été en grande partie, ou même en totalité dégradé au cours de l'hydrolyse des caséines et des opérations effectuées avant dosage, et les quantités trouvées n'en représenteraient qu'une partie, ou proviendraient d'autres polysaccharides, ou même des cellules (fraction insoluble) et de leur lyse partielle (fraction soluble). La préparation de Pronase utilisée ne peut être mise en cause car elle était dépourvue d'activité glucolytique et a d'ailleurs servi à l'obtention d'un autre polysaccharide (CERNING et al., 1986). Mais il a déjà été montré qu'un polysaccharide produit par L. bifidus pouvait être dépolymérisé en aérobiose en présence des cellules viables (WANG et al., 1963);

- ou bien le composé responsable n'est pas un polysaccharide mais une (ou des) glycoprotéine comme cela a été montré chez des $S$. cremoris visqueux isolés du vilii (Macura et Townsley, 1984). Si c'est le cas, ce composé est certainement en grande partie hydrolysé par le traitement de digestion des caséines et il serait certainement très difficile de le doser et de l'isoler directement à partir de cultures sur lait ;

- ou bien enfin il reste l'hypothèse que les modifications de propriété du gel ne sont pas dues à la présence de ces types de composés mais à une autre cause. D'après nos résultats, il ne s'agirait cependant pas d'une modification des caséines par des enzymes telles que des protéases, exocellulaires ou liées aux enveloppes, ce type d'enzyme n'a d'ailleurs pas été mis en évidence jusqu'à maintenant chez $S$. thermophilus (Desmazeaud, 1983).

Reçu le 8 octobre 1986. Accepté pour publication le 6 décembre 1986.

\section{Références bibliographiques}

Bouillanne C., Desmazeaud M.J., 1980. Etude de quelques caractères de souches de Streptococcus thermophilus utilisées en fabrication de yoghourt et proposition d'une méthode de classement. Lait, 60, 458-473.

BROOKER B.E., 1979. Electron microscopy of the dextrans produced by lactic acid bacteria. In : Microbial polysaccharides and polysaccharases, ed. Berkeley et al., Academic Press, London.

Cerning J., Boullanne C., Desmazeaud M,J., Landon M., 1986. Isolation and characterization of exocellular polysaccharide produced by Lactobacillus bulgaricus. Biotechnol. Lett., 9, 625628.

Dain J.A., Neal A.L., Seely H.W., 1956. The effect of carbon dioxyde on polysaccharide production by Streptococcus bovis. J. Bacteriol., 72, 209-213.

Desmazeaud M.J., 1983. L'état des connaissances en matière de nutrition des bactéries lactiques. Lait, 63, 267-273.

Dubois M., Gilles K.A., Hamilton J.K., Rebers P.A., Smith F., 1956. Colorimetric method for determination of sugars and related substances. Anal. Chem., 28, 350-356.

Dunican L.K., SeEly H.W., 1965. Extracellular polysaccharide synthesis by members of the genus Lactobacillus. Conditions for formation and accumulation. J. Gen Microbiol., 40, 297-308. 
Elliker P.R., Anderson A.W., Hannesson G., 1956. An agar culture medium for lactic streptococci and lactobacilli. J. Dairy Sci., 39, 1611-1612.

Fukushima K., TAKada, Motoda R., IKeda T., 1982. Independence of water-insoluble glucan synthesis and adherence of Streptococcus mutans to smooth surfaces. FEBS Lett., 149, 299303.

Galesloot T.E., Hassing F., 1973. Handhaven van de stijmstofproduktie van yoghurtculturen. Ned. Inst. Zuivelonderz. NIZO Mededalingen, 7, 57-62.

Groux M., 1973. Critical observations of yoghurt manufacture with reference to protein breakdown. Schweizt. Milchzeit., 99, 18-20.

LAEMmLi U.K., 1970. Cleavage of structural proteins during the assembly of head of bacteriophage T4. Nature, 227, 680-685.

La Rivière J.W.M., Kooiman P., Schmidt K., 1967. Kefiran, a novel polysaccharide produced in the kefir grain by Lactobacillus brevis. Arch. Mikrobiol., 59, 269-278.

Macura D., TownSLey P.M., 1984. Scandinavian ropy milk. Identification and characterization of endogenous ropy lactic streptococci and their extracellular excretion. J. Dairy Sci., 67, 735744.

Manca De Nadra J.C., Strasser De Saad A.M., Pesce De Ruiz Holgado A.A., Oliver G., 1985. Extracellular polysaccharide production by Lactobacillus bulgaricus CRL 420. Milchwissenschaft, 40, 409-411.

Martens R., 1972. Influence de quelques facteurs sur la consistance du yaourt brassé. Rev. Agric., $25,461-480$.

Niven C.F., Smiley J.R., Sherman J.M., 1941. The production of large amount of a polysaccharide by Streptococcus salivarius. J. Bacteriol., 41, 479-484.

Niven C.F., Kiziuta J.Z., White J.C., 1946. Synthesis of a polysaccharide from sucrose by Streptococcus SBE. J. Bacteriol,, 51, 711-716.

Oda M., Hasegawa H., Komatsu S., Kambe M., Tsuchiya F., 1983. Anti-tumor polysaccharide from Lactobacillus sp. Agric. Biol. Chem., 47, 1623-1625.

Ranganathan B., Chander H., Tiwari M.P., 1979. Studies on factors affecting ropiness in $S$. lactis. Milchwissenschaft, 34, 333-334.

SchellhaAss S.C., 1983. Characterization of exocellular slime produced by bacterial starter culture used in the manufacture of fermented dairy products. Ph. D. Thesis, University of Minnesota, L.S.A.

Slodki M.E., Cadmus, 1978. Production of microbial polysaccharides. Adv. Appl. Microbiol., 23, $19-54$.

Sutherland I.W., 1982. Biosynthesis of microbial exopolysaccharides. Adv. Microbiol., 23, 79-150.

TAmime A.Y., Robinson R.K., 1978. Some aspects of the production of a concentrated yoghurt (labneh) popular in the Middle East. Milchwissenschaft, 33, 209-212.

Trieu-Cuot P., Gripon J.C., 1981. Electrofocusing and two-dimensional electrophoresis of bovine caseins. J. Dairy Res., 48, 303-310.

Tsuchiya F., Miyazawa K., Kanbe M., Oda M., Ebisawa N., 1981. Polysaccharide MPS-80 de haute masse moléculaire et sa préparation. Brevet $\mathrm{n}^{\circ} 8122548$, I.N.P.I., Paris.

Uriel J., 1966. Méthode d'électrophorèse dans des gels de polyacrylamide agarose. Bull. Soc. Chim. Biol., 48, 969-982.

VAnderpoorten R., Waes G., 1972. Etude des propriétés de quelques cultures de yoghourt. Rev. Agric., 25, 101-114.

Wang M., Steers E., Norris R.F., 1963. Extracellular polysăccharide of mucoïd Lactobacillus bifidus. J. Bacteriol., 86, 898-903. 